\title{
A Cultura Alimentar e os Reflexos de um Aprisionamento da Condição Social ${ }^{1}$
}

\author{
La Cultura Alimentaria y los Reflejos de un Encarcelamiento de la \\ Condición Social \\ The Food Culture and the Reflexes of an Imprisonment of the Social \\ Condition
}

\author{
Ana Carolina Einsfeld Mattos ${ }^{2}$ \\ Victória Santos de Azevedo ${ }^{3}$
}

\begin{abstract}
Resumo
Primeiramente: Comer é um ato político! Mas somente para quem pode escolher o que comer! Segundo, a alimentação é um direito humano. Comer é uma atividade humana central, porque cedo se torna a esfera onde se permite alguma escolha. O pensamento antropológico da alimentação diz que o comportamento relativo à comida revela repetidamente a cultura. Comer! Comer é natural, é cotidiano. O que, como e quando comemos caracteriza respostas culturais a contextos sociais, políticos, econômicos, étnicos, ambientais e morais. A globalização respinga uma nova forma de constituir alimentação. Os fast- foods acompanhado do marketing das grandes empresas transnacionais tem contribuído para essa mudança nas estruturas alimentares. $\mathrm{O}$ fio condutor entre alimentação e cultura parece limitar-se ao nível biológico, que coloca o alimento com foco nos nutrientes, porém um enfoque nas ciências sociais amplia o olhar para a relação do alimento enquanto componente de vida e do viver em sociedade. Assim, os hábitos e práticas alimentares produzidos historicamente se transformam em hábitos culturais que integram o viver coletivo. Considera-se também que existem hábitos e práticas alimentares advindas de um sistema capitalista e que por tanto não constituem um caráter homogêneo. Há diferentes modos de estabelecer a alimentação e a comida, a estratificação da mesa e do gosto produz efeitos sobre a condição social da pessoa que reduz a possibilidade de escolha alimentar em uma espécie de aprisionamento da condição social. A preferência alimentar transmite mais que a utilização do alimento, um estabelecimento de uma preparação culinária, temperada, saboreada, estabelece identidade social. Alimenta-se e alimentar ao próximo é uma das maiores riquezas quando pensamos em constituição de relações sociais. O guia Alimentar para a população Brasileira constitui uma das estratégias para promoção da alimentação adequada, visa o resgate da cultura alimentar e faz parte da Política Nacional de Alimentação e Nutrição.
\end{abstract}

Palavras-Chave: Cultura alimentar; Globalização alimentar; Alimentação contemporânea; Resgate da Cultura Alimentar.

\section{Resumen}

Primero: Comer es un acto político! ¡Pero solamente para quién puede elegir qué comer! Segundo, la alimentación es un derecho humano. Comer es una actividad humana central, porque pronto se convierte en la

\footnotetext{
${ }^{1}$ Artigo apresentado no II Seminário Latino-Americano de Estudos em Cultura - SEMLACult em Foz do Iguaçu/PR, Brasil, 2018.

${ }^{2}$ Mestranda em Ciências Sociais; nutricionista e especialista em saúde mental pela Universidade do Vale do Rio dos Sinos - UNISINOS; São Leopoldo, Rio Grande do Sul, Brasil; anamattos50@gmail.com.
}

\footnotetext{
${ }^{3}$ Mestranda em Ciências Sociais e advogada pela Universidade do Vale do Rio dos Sinos - UNISINOS; São Leopoldo, Rio Grande do Sul, Brasil; vics.azevedo@gmail.com.
} 
esfera donde se permite alguna elección. El pensamiento antropológico de la alimentación dice que el comportamiento relativo a la comida revela repetidamente la cultura. Comer! Comer es natural, es cotidiano. Lo que, como y cuando comemos caracteriza respuestas culturales a contextos sociales, políticos, económicos, étnicos, ambientales y morales. La globalización respinga una nueva forma de constituir alimentación. Los fastfoods acompañados del marketing de las grandes empresas transnacionales han contribuido, para ese cambio en las estructuras alimentarias. El hilo conductor entre alimentación y cultura parece limitarse al nivel biológico, que coloca el alimento con foco en los nutrientes, pero un enfoque en las ciencias sociales amplía la mirada a la relación del alimento como componente de vida y del vivir en sociedad. Así, los hábitos y prácticas alimentarios producidos históricamente se transforman en hábitos culturales que integran el vivir colectivo. Se considera también que existen hábitos y prácticas alimentarias provenientes de un sistema capitalista y que por tanto no constituyen un carácter homogéneo. Hay diferentes modos de establecer la alimentación y la comida, la estratificación de la mesa y del gusto produce efectos sobre la condición social de la persona en que se reduce la posibilidad de elección alimentaria en una especie de encarcelamiento de la condición social. La preferencia alimentaria transmite más que la utilización del alimento, un establecimiento de una preparación culinaria, templada, saboreada y establece identidad social. Se alimenta y alimenta al prójimo es una de las mayores riquezas cuando pensamos en la constitución de relaciones sociales. La guía Alimentaria para la población brasileña constituye una de las estrategias para promover la alimentación adecuada, busca el rescate de la cultura alimentaria y forma parte de la Política Nacional de Alimentación y Nutrición.

Palabras claves: Cultura alimentaria; Globalización alimentaria; Alimentación contemporánea; Rescate de la Cultura Alimentaria.

\begin{abstract}
First: Eating is a political act! But only for those who can choose what to eat! Second, food is a human right. Eating is a central human activity, because soon it becomes the sphere where some choice is allowed. Anthropological thinking of food says that behavior relating to food repeatedly reveals culture. Eat! Eating is natural, everyday. What, how and when we eat characterizes cultural responses to social, political, economic, ethnic, environmental and moral contexts. Globalization establishes a new way of feeding. Fast-foods accompanied by the marketing of large transnational corporations have contributed, for this change in food structures. The guiding line between food and culture seems to be limited to the biological level, which places food with a focus on nutrients, but a focus on the social sciences widens the look at the relationship of food as a component of life and of living in society. Thus, habits and food practices produced historically become cultural habits that integrate the collective life. It is also considered that there are habits and alimentary practices coming from a capitalist system and that therefore do not constitute a homogenous character. There are different ways of establishing food, the stratification of the table and the taste produces effects on the social condition of the person in which the possibility of food choice is reduced in a kind of imprisonment of the social condition. The food preference transmits more than the use of the food, an establishment of a culinary preparation, tempered, savored and establishes social identity. Feeding oneself and nourishing others is one of the greatest riches when we think of social relationships. The Food Guide for the Brazilian population is one of the strategies to promote adequate food, it aims to rescue food culture and is part of the National Food and Nutrition Policy.
\end{abstract}

Keywords: Food culture; Food globalization; Contemporary food; Rescue of the Food Culture.

\title{
1. Introdução
}

Dois pontos críticos a serem levantados inicialmente quanto à relação da alimentação no atual contexto de organização social capitalista. Primeiro, comer é de fato um ato político! Mas só é um ato político para quem pode escolher o que vai comer! Segundo, comer é um direito humano social e fundamental (SIDEKUM et. al., 2016). A alimentação é em suma, a luta contra a fome, e nem sempre essa luta se manteve com índices positivos para a humanidade. A fome está presente no passado da história da civilização, como também nos 
dias atuais (CARNEIRO, 2003). Os aspectos que envolvem a alimentação é sempre um tema atual e a fome no Brasil enfrenta uma onda de retorno!

O Direito Humano a Alimentação Adequada (DHAA) consiste em um direito humano fundamental social. Corresponde universalmente a todos os seres humanos, e de forma estatal assegura que os alimentos estejam disponíveis a todos e a todas, se entendendo que a falta desse alimento afeta diretamente a dignidade da pessoa humana (SIDEKUM et. al., 2016).

Qualquer programa social que tenha como foco o recurso da disponibilidade da boa vontade solidária e voluntária não consegue garantir continuidade e muito menos superação da fome (PINTO, 2005). Algo nesse processo não deu certo, e a solidariedade hoje é um dos pontos que torna a alimentação mais relacionada à caridade, assistencialismo, e benevolência alheia do que uma missão de posse de direito. A fome está presente e nunca conseguiu ser superada, hoje, século XXI, ano de 2018, o Brasil retorna ao mapa da fome!

A partir da Constituição de 1988 diversos direitos importantes tiveram legitimação, contudo, nenhuns destes direitos, escritos no papel, foram suficientes para que por parte estatal se criassem ações de enfrentamento da exclusão social por parte da população vulnerável. Garantir acesso à cidadania tornasse papel central com a garantia de direitos, e a alimentação como posse de direito ao enfrentamento da fome é ressente no País. O Brasil tem mantido historicamente índices elevados de indivíduos abaixo da linha da pobreza, ou seja, em situação de fome (PINTO, 2005). A estrutura capitalista é relacional para tal situação.

A fome no Brasil é antes de tudo uma luta histórica, e a fome coletiva é generalizada. Geograficamente um fenômeno universal, a fome é uma das mais graves consequências da miséria, e neste estado de fome vegetam diversas populações (CASTRO, 1984). Eis que dentro deste emaranhado estrutural das sociedades contemporâneas, é notória uma espécie de aprisionamento dentro das condições sociais de vida, e que este estabelece uma forma cultural de estabelecer a alimentação.

Comer é uma atividade humana central não só por sua frequência, constante e necessária, mas também porque cedo se torna a esfera onde se permite alguma escolha. $\mathrm{O}$ pensamento antropológico da alimentação diz que o comportamento relativo à comida revela repetidamente a cultura em que cada um está inserido. Nossos filhos são treinados de acordo com isso. O aprendizado que apresenta características como requinte pessoal, destreza 
manual, cooperação e compartilhamento, restrição e reciprocidade, é atribuído à socialização alimentar das crianças por diferentes sociedades (MINTZ, 2001).

Comer! Comer é usual, é cotidiano, e é cultural! Torna-se centro na vida humana. Para além do biológico, comer exige um lugar, um tempo, uma companhia, ou não, comemos para celebrar, para confortar, por prazer, por simplesmente querer, ou precisar. O que se come, como se come e quando comemos caracteriza respostas culturais a contextos sociais, políticos, econômicos, étnicos, ambientais e morais (MOTA e PENA, 2014).

O alimento enquanto resgate cultural está direcionado ao fazer alimentação para além do nutriente, que constitui uma forma de prazer sensorial, abrange um ritual, e com isso vincula significados. Assim nossa presunção ao trazer o termo "hábito alimentar" não se limita a uma ação mecânica de manutenção do corpo, mas sim uma atitude significativa do papel que a alimentação exerce na vida.

E como pensar a alimentação e a cultura alimentar nesse desmantelamento de princípios que nos coloca frente a uma estrutura de sociedade em que as pessoas precisam utilizar o seu tempo para produzir recursos sob uma constante exploração? Como pensar alimentação e cultura em contraste com uma estrutura que coloca diversos indivíduos cara a cara com a fome? Como valorizar o que de fato é uma alimentação saudável quando uma estrutura social capitalista não permite sequer uma escolha alimentar?

Cria-se uma nova forma de cultura alimentar dentro das limitações sociais, contudo, como cita Pollan (2010), "Não coma nada que sua avó não reconheceria como comida", o que traz a ideia em recorrer aos primórdios da nossa constituição enquanto seres e valorizar na atualidade o resgate da cultura alimentar, entretanto, visando atentar para o meio social que estamos inseridos e as possibilidades e obstáculos para o tal resgate da cultura alimentar. Alimenta-se e alimentar ao próximo é uma das maiores riquezas quando pensamos em constituição de relações sociais, eis que comer é de fato importante, pois além de necessidade básica vital, nos torna pertencente de um tecido social.

Visto isso, neste artigo temos o objetivo de contextualizar os aspectos relacionados à alimentação contemporânea com um enfoque na defesa de um necessário resgate da cultura alimentar, para isso é necessário discutir a forma como se concretiza a alimentação na sua totalidade, assim como questões que estão ao redor do assunto, como a autonomia de escolha alimentar e a estrutura capitalista de sociedade ao qual pertencemos. 


\section{Escolha Alimentar e o Aprisionamento condição Social}

Ao relacionar a alimentação aos aspectos da autonomia nas escolhas alimentares, existem dois pontos que precisam ser levados em consideração, à existência de uma dimensão relacional entre fatores econômicos, sociais e culturais. É fácil pensar que alguém ao escolher o seu alimento em um aspecto individual colocará em prática seus conhecimentos sobre alimentação, suas percepções sobre o que concretiza uma alimentação saudável e suas preferências alimentares (BRASIL, 2012). Contudo é necessário levar em consideração as possibilidades de escolha alimentar no que tange o acesso e as condições para tal aquisição.

Formou-se o mito da riqueza, ou melhor, do crescimento da riqueza (...) maximizando a produtividade da mão de obra e do capital, chegar-se-ia a promover a generalização do progresso e da riqueza (CASTRO, p. 88, 1984).

A distribuição da riqueza guiada pelo próprio mercado se absorve como um processo usual e a pobreza como algo destinado a grupos incapazes de garantir uma inserção, o que é um engano, pois a "mão visível" neste caso dos dominantes e privilegiados sempre monopolizou os benefícios deixando a miséria aos grupos marginalizados (MAGALHÃES, 1997).

O acesso diário a alimentação, ou a posse de alimentação, depende nas economias mercantis de poder aquisitivo, isto é, ter renda para acessar determinada alimentação, uma parcela significativa da população brasileira se mantém com rendimentos tão baixos que as coloca em linha de pobreza e de insegurança alimentar (HOFFMANN, 1995).

Há diferentes modos de estabelecer a alimentação e a comida, Freitas et. al. (2008) esclarecem que, a estratificação da mesa e do gosto produz efeitos sobre a condição social da pessoa em que se reduz a possibilidade de escolha alimentar, o que constitui diferenças no estabelecimento dos hábitos alimentares. A redução da escolha inclui-se em uma espécie de aprisionamento da condição social, onde a partir do que tenho acesso se constitui a alimentação e todas as suas facetas. A preferência alimentar transmite mais que a utilização do alimento, pois quando este percorre um estabelecimento de uma comida, de uma preparação culinária, mais que alimento, neste caso a comida é temperada, saboreada, estabelece identidade social, e absorve o estabelecimento de uma cultura alimentar.

Ao transcorrer para os aspectos que envolvem a pobreza, geralmente é fácil relacionar com a noção de renda, baseada no ganho para realização das necessidades dos indivíduos, e 
ao associar a fome crônica temos um determinante, a alimentação e seu custo. A base para determinação da linha da pobreza é definida em vias de insegurança alimentar (MONTEIRO, 2003).

Por outro viés na extensão da urbanização onde as pessoas se aglomeram, na industrialização dos produtos alimentares e em seu marketing de oferta, e na mídia imposta na implantação dos produtos ora colocados no mercado como obrigatoriedade de aquisição, ocorre um movimento de transferência de cultura alimentar a um contexto impositivo, onde todos são induzidos a adequarem a nova moda e onda proposta (LEONARDO, 2009).

Outro aspecto importante a ser discutido são alguns valores atribuídos à modernidade como a pressa, comodidade, praticidade, e que aliados à dominação das grandes empresas alimentícias contribuem para os novos comportamentos alimentares contemporâneos, sobretudo no meio urbano, que se caracterizam pelos alimentos rápidos e estes normalmente se encontram dentro de embalagens comercializadas (OLIVEIRA e FREITAS, 2008).

Carneiro (2003) ressalta um irônico paradoxo relacionado à modernização da alimentação, uma condição da contemporaneidade que de forma usual coloca uma alta escala de produção de alimentos em contra partida a ocorrência enorme de pessoas famintas no mundo de hoje. Essa relação da disponibilidade alimentar em crescente aparência coloca um limite na aquisição desse disponível.

A fome- eis um problema tão velho quanto à própria vida (CASTRO, p.5, 1984).

\section{Dialogando o Resgate da Cultura Alimentar}

A ocidentalização da dieta e a globalização dos hábitos alimentares respinga uma nova forma de constituir a alimentação e por consequência a nutrição. Os fast-foods acompanhado da mídia e do marketing das grandes empresas transnacionais aliado a transformações na produção e no processamento dos alimentos ocorridas nas últimas três décadas, tem contribuído e muito, para essa mudança nas estruturas da alimentação (CARNEIRO, 2003).

A discussão entre o fio condutor entre alimentação e cultura parece limitar-se ao nível biológico, que coloca o alimento como único e exclusivo foco na disponibilidade de nutrientes necessários para a manutenção da vida, porém um enfoque nas ciências sociais amplia o olhar para a relação do alimento enquanto componente de vida e do viver em sociedade (PACHECO, 2008). 
O alimento constitui uma categoria histórica e a dinâmica que envolve os processos sociais representam referências de práticas alimentares, pois os padrões de permanência e mudanças dos hábitos acompanham essas dinâmicas. Os alimentos não são somente alimentos. Alimentar-se é um ato nutricional, comer é um ato social, pois constitui atitudes ligadas aos usos, costumes, protocolos, condutas e situações individuais e coletivas na representação da alimentação (SANTOS, 2005).

Assim, os hábitos e práticas alimentares produzidos historicamente se transformam em hábitos culturais que integram o viver coletivo. Considera-se também que existem hábitos e praticas alimentares advindas do sistema capitalista e que, portanto não constituem um caráter homogêneo, pois não podem ser transformados em prática alimentar por todos os indivíduos, mesmo sendo desejo de todos (PACHECO 2008). Uma espécie de retrocesso político que transmite valorização do lucro pela alimentação e que (des) configura os processos culturais relacionados à alimentação.

O guia Alimentar para a população Brasileira constitui em uma das estratégias para implementação da diretriz da promoção da alimentação adequada e saudável que faz parte da Política Nacional de Alimentação e Nutrição (PNAN). O guia alimentar fornece um incentivo legitimado na defesa da alimentação e do resgate da cultura alimentar (BRASIL, 2015).

Outra ação que merece valorização quando levantamos à bandeira da defesa da alimentação saudável, do resgate a cultura alimentar, e do protagonismo dos indivíduos que compõe a sociedade, são as hortas coletivas/ comunitárias, que trazem o retorno do "comer alimento", e não a limitação social na aquisição de produtos alimentícios. Alimentos que não geram custos e não possuem marcas, produzidos por mãos coletivas/ individuais e destinados ao autoconsumo, assim como possibilidade de autossustento e emancipação social.

\section{Conclusões}

A alimentação no contexto da vida, abarca diferentes correntes de pensamento, que percorrem desde uma modernização contemporânea da alimentação, com um leque de opções alimentares em crescente aumento, até uma crítica a desconstrução de raízes alimentares no decorrer dos processos históricos.

As grandes empresas de produtos alimentícios controlam o mercado de alimentos e promovem a transição da alimentação natural para uma proposta de alimentação rápida e industrial. Em sentido contrário, existe uma corrente que procura dialogar uma regressão 
positiva desses processos de modificação alimentar que o mundo moderno necessita. O tempo limitado, o imperialismo do individualismo sobre o coletivo e um fluxo de aparições midiáticas contribui para uma aceleração da vida e da alimentação.

Nesta perspectiva, a importância da luta por condições para que cada pessoa possa definir seu projeto de vida. Uma espécie de empoderamento dos sujeitos, decisões e condições para assumir seus projetos de vida pessoal e coletiva, na construção da vida digna, e que cada ser humano expresse suas diferenças e possuam capacidade de se relacionar com seu meio de vida, dando um sentido para sua própria realidade (GÁNDARA, 2013).

Em meio às estruturas de sociedade, as escolhas alimentares, embora de forma autônomas, são acompanhadas de diversas influências contextuais e também de uma limitação nas possibilidades de cada indivíduo. As referencias de escolhas abrangem um contexto político, econômico, religioso, social e também cultural.

Por fim, o art. $6^{\circ}$ da constituição, cita os direitos fundamentais e declara a alimentação como um direito social, na função de distribuir a riqueza, para fins não apenas de eliminar, por benevolência, a pobreza, mas para compor o projeto de uma sociedade na qual todos possam, efetivamente, adquirir, em sua significação máxima o sentido de cidadania.

Visto isso, a valorização da alimentação e da nutrição na busca de um resgate cultural do comer torna uma defesa importante a ser enfrentada em épocas atuais. O comer cercado de significados que garantem uma identidade social, assim como o reconhecimento do alimento saudável enfrentam crises. É preciso valorizar iniciativas que enfrentam essas demandas e legitimar ações, como é o caso das hortas urbanas individuais e comunitárias. A alimentação carece de incentivos para que de fato alcance um estágio de segurança alimentar e concretização do Direito Humano à Alimentação Adequada.

\section{Referências}

BRASIL. Ministério do Desenvolvimento Social e Combate à Fome. Marco de Referência de Educação Alimentar e Nutricional para as políticas Públicas. - Brasília, DF: MDS; Secretaria Nacional de Segurança Alimentar e Nutricional; 2012. (obra completa)

BRASIL. Ministério da Saúde. Secretaria de Atenção à Saúde. Departamento de Atenção Básica. Guia Alimentar Para a População Brasileira. Ministério da Saúde, Secretaria de Atenção à Saúde, Departamento de Atenção Básica- 2. Ed., 2 reimpr.- Brasília: Ministério da Saúde, 2015. (obra completa) 
CARNEIRO, H. Comida e Sociedade: Uma história da alimentação. Rio de Janeiro: Campus, 2003. (obra completa)

CASTRO, J. Geografia da fome: o dilema brasileiro: pão ou aço. Rio de Janeiro: Edições Antares, 1984. (obra completa)

FREITAS, M. C. S.; PENA, P. G. L.; FONTES, G. A. V.; SILVA, D. O.; SANTOS, L. A.; MELLO, A. O.; ALMEIDA, M. D. Uma leitura Humanista da nutrição. In: FREITAS, M. C. S.; FONTES, G. A. V.; OLIVEIRA, N.(org.). Escritas e Narrativas sobre Alimentação e Cultura. Salvador: EDUFBA, 2008. (Capítulo de Livro)

GÁNDARA, Manuel. Derechos humanos y capitalismo: reflexiones en perspectiva sociohistórica. REDHES - Revista de Derechos Humanos y Estudios Sociales $\mathrm{n}^{\mathrm{o}}$.10, año V, juliodiciembre 2013. P. 135-156. Disponível em:

http://www.derecho.uaslp.mx/Documents/Revista\%20REDHES/N\%C3\%BAmero\%2010/Red hes10-06.pdf. Acesso em: 22 dezembro. 2018. (Artigo em Periódico Digital)

HOFFMANN, R. Pobreza, insegurança alimentar e desnutrição no Brasil. Estudos Avançados, v. 9, n. 24, 1995. Disponível em:

http://www.scielo.br/pdf/ea/v9n24/v9n24a07.pdf. Acesso em: 22 dezembro. 2018. (Artigo em Periódico Digital)

LEONARDO M. Antropologia da Alimentação. Revista Antropos - Volume 3, Ano 2, Dezembro de 2009. Disponível em: http://revista.antropos.com.br/downloads/dez2009/Artigo\%201\%20-

$\% 20$ Anntropologia\%20da\%20Alimenta\%E7\%E3o\%20-\%20Maria\%20Leonardo.pdf. Acesso em: 17 maio. 2018. (Artigo em Periódico Digital)

MAGALHÃES, R. Fome: uma (re) leitura de Josué de Castro. Rio de Janeiro: Fiocruz, 1997. (obra completa)

MINTZ, S. W. Comida e antropologia: Uma breve revisão. RBCS Vol. 16 n 47 outubro/2001. Disponível em: http://www.scielo.br/pdf/rbcsoc/v16n47/7718. Acesso em: 17 maio. 2018. (Artigo em Periódico Digital)

MONTEIRO, C. A. A dimensão da pobreza, da desnutrição e da fome no Brasil. Estudos Avançados, v. 17, n. 48, 2003. Disponível em:<http://www.scielo.br/pdf/ea/v17n48/v17n48a02.pdf. Acesso em: 22 dezembro. 2018. (Artigo em Periódico Digital)

MOTA, S. E. C.; PENA, G. L. Significados socioculturais da alimentação em uma comunidade de Marisqueiras no Recôncavo Baiano. In: FREITAS, M. C. S.; SILVA, D. O. (org.). Narrativas sobre o comer no mundo da Vida. Salvador: EDUFBA, 2014. (capítulo de Livro)

OLIVEIRA,N.; FREITAS, M. C. S. Fast-Food: Um aspecto da modernidade alimentar. In: FREITAS, M. C. S.; FONTES, G. A. V.; OLIVEIRA, N.(org.). Escritas e Narrativas sobre Alimentação e Cultura. Salvador: EDUFBA, 2008. (capítulo de Livro)

PACHECO, S. S. M. O hábito alimentar enquanto comportamento culturalmente produzido. Im: FREITAS, M. C. S.; FONTES, G. A. V.; OLIVEIRA, N.(org.). Escritas e Narrativas sobre Alimentação e Cultura. Salvador: EDUFBA, 2008. (capítulo de Livro) 
PINTO, C. R. J. A sociedade civil e a luta contra a fome no Brasil (1993- 2003). Sociedade e Estado, Brasília, v. 20, n. 1, p. 195-228, jan.-abr., 2005. Disponível em:

http://www.scielo.br/pdf/se/v20n1/v20n1a09.pdf. Acesso em: 22 dezembro. 2018. (Artigo em Periódico Digital)

POLLAN, M. Regras da Comida: Um Manual da Sabedoria Alimentar. Tradução de Adalgisa Campos da Silva. - Rio de Janeiro: Intrínseca, 2010. (obra completa)

SANTOS, C. R. A. A alimentação e seu lugar na história: os tempos da memória gustativa. Editora UFPR História: Questões \& Debates, Curitiba, n. 42, p. 11-31, 2005. Disponível em: https://revistas.ufpr.br/\%EE\%80\%80historia\%EE\%80\%81/article/viewFile/4643/3797.

Acesso em: 18 maio. 2018. (Artigo em Periódico Digital)

SIDEKUM, A.; WOLKMER, A. C.; RADAELLI, S. M. Enciclopédia Latino-americana dos Direitos Humanos. Blumenau: Edifurb; Nova Petrópolis: Nova Harmonia, 2016. (obra completa). 